Pag.: 37 - 46

\title{
ENFERMAGEM DE REABILITAÇÃO EM TRANSPLANTE CARDÍACO (ERTXC) - APRESENTAÇÃO DE PROJETO DE INTERVENÇÃO
}

\section{REHABILITATION NURSING FOR HEART TRANSPLANT (RN4HTX) - INTERVENTION PROJECT PRESENTATION}

\section{ENFERMERÍA DE REHABILITACIÓN EN TRASPLANTE CARDÍACO (ERTXC) - PRESENTACIÓN DE PROYECTO DE INTERVENCIÓN}

\author{
Maria Loureiro ${ }^{(1)}$, Marta Costa ${ }^{(2)}$, Emília Sola ${ }^{(3)}$, Gonçalo Coutinho ${ }^{(4)}$, Maria Manuela Martins ${ }^{(5)}$, André Novo ${ }^{(6)}$, \\ (1) Instituto de Ciências Biomédicas Abel Salazar da Universidade do Porto; CINTESIS:NursID, Porto; Centro Hospitalar e Universitário de Coimbra, Portugal; \\ (2) Centro Hospitalar e Universitário de Coimbra, Coimbra, Portugal; (3) Centro Hospitalar e Universitário de Coimbra, Coimbra, Portugal; (4) Centro \\ Hospitalar e Universitário de Coimbra; Faculdade de Medicina de Coimbra, Coimbra, Portugal; (5) Escola Superior de Enfermagem do Porto (ESEP), \\ CINTESIS:NursID, Porto, Portugal; (6) CINTESIS:NursID, Porto; Instituto Politécnico de Bragança, Bragança, Portugal;
}

\section{Descritores}

Reabilitação Cardíaca Transplante de Coração Cuidadores

Enfermagem de Reabilitação

\section{Resumo}

Introdução: A Reabilitação Cardíaca (RC) é uma intervenção multifactorial, geralmente composta por exercício físico estruturado, apoio psicológico e educação para a saúde. A estruturação nas diferentes fases de RC para esta população específica não está profundamente estudada, existindo lacunas na evidência científica relativamente aos ganhos em saúde decorrentes de programas de RC. A literatura relativa ao cuidador/família é também escassa. 0 objetivo deste estudo é identificar em que domínios é que os enfermeiros especialistas de reabilitação poderão intervir de forma a desenvolver ganhos em saúde de pessoas transplantadas cardíacas e seu cuidador/família.

Método: Para a operacionalização desta intervenção será utilizado o The Quality Health Outcomes Model, de Doran, na componente de resultados sensíveis aos cuidados de enfermagem. Serão efetuados de forma concorrente 2 estudos, um direcionado à reabilitação cardíaca da pessoa transplantada (fases 1,2 e 3 ) e outro com enfoque na reabilitação cardíaca do cuidador/família. As metodologias utilizadas serão a scoping review, o relato de caso, o estudo exploratório e o estudo clínico randomizado (com grupo de intervenção e grupo de controlo).

Discussão/Resultados Esperados: Será construído e implementado o programa Enfermagem de Reabilitacão em Transplante Cardíaco (ERtxC), nas suas diferentes fases, durante os anos de 2021 e 2022. Estima-se que o programa possa ser acedido por 150 pessoas transplantadas cardíacas e respetivas familias/cuidador.

Conclusão: No final do ano de 2022 estima-se ter chegado ao final deste programa, com respetiva avaliação dos ganhos em saúde. Após validação deste programa, tentar-se-á criar em definitivo esta oferta de forma sistemática para todas pessoas submetidas a transplante cardíaco, em Portugal.

\section{Abstract}

Introduction: Cardiac Rehabilitation (CR) is a multifactorial intervention, usually composed of structured physical exercise, psychological support and health education. The structuring in the different phases of CR for this specific population has not been deeply studied, with gaps in the scientific evidence regarding health gains resulting from CR programs. The caregiver / family literature is also scarce. The aim of this study is to identify in which domains rehabilitation nurses can intervene in order to develop health gains in heart transplant recipients and their caregivers/family.

Method: To make this intervention operational, Doran's The Quality Health Outcomes Model will be used, in the component of results sensitive to nursing care. Two studies will be carried out 
concurrently, one aimed at the cardiac rehabilitation of the transplanted person (phases 1, 2 and 3 ) and the other focusing on the cardiac rehabilitation of the caregiver / family. The methodologies used will be the scoping review, the case report, the exploratory study and the randomized clinical study (with intervention group and control group).

Discussion/Expected Results: The Rehabilitation Nursing in Cardiac Transplant Program (ERtxC) will be built and implemented, in its different phases, during the years 2021 and 2022. It is estimated that the program can be accessed by 150 heart transplant patients and their respective families / caregiver.

Conclusion: At the end of the year 2022, it is estimated to have reached the end of this program, with the respective assessment of health gains. After validation of this program, an attempt will be made to permanently create this offer in a systematic way for all people undergoing heart transplantation in Portugal.

\section{Descriptores}

Rehabilitación Cardiaca Trasplante de Corazón Cuidadores

Enfermería de Rehabilitación

\section{Resumen}

Introducción: La rehabilitación cardíaca $(\mathrm{RC})$ es una intervención multifactorial, generalmente compuesta por ejercicio físico estructurado, apoyo psicológico y educación para la salud. La estructuración en las diferentes fases de la RC para esta población específica no ha sido profundamente estudiada, y existen lagunas en la evidencia científica sobre los beneficios en salud resultantes de los programas de RC. La literatura sobre cuidadores / familiares también es escasa. El objetivo de este estudio es identificar en qué dominios pueden intervenir las enfermeras especializadas en rehabilitación para desarrollar beneficios para la salud de los receptores de trasplantes de corazón y sus cuidadores / familiares.

Método: Para hacer operativa esta intervención, se utilizará el Modelo de Resultados de Calidad en Salud de Doran, en el componente de resultados sensibles al cuidado de enfermería. Se realizarán dos estudios de forma simultánea, uno dirigido a la rehabilitación cardíaca del trasplantado (fases 1,2 y 3 ) y el otro centrado en la rehabilitación cardíaca del cuidador / familia. Las metodologías utilizadas serán la revisión de alcance, el caso clínico, el estudio exploratorio y el estudio clínico aleatorizado (con grupo de intervención y grupo de control).

Discusión/Resultados esperados: El Programa de Enfermería de Rehabilitación en Trasplante Cardíaco (ERtxC) se construirá e implementará, en sus diferentes fases, durante los años 2021 y 2022. Se estima que el programa puede ser accedido por 150 pacientes trasplantados cardíacos y sus respectivos familias / cuidador.

Conclusión: Para fines del año 2022, se estima haber llegado al final de este programa, con la respectiva evaluación de los logros en salud. Una vez validado este programa, se intentará crear de forma permanente esta oferta de forma sistemática para todas las personas sometidas a trasplante de corazón, en Portugal.

\section{INTRODUÇÃO}

A insuficiência cardíaca refratária é condição terminal de diversas cardiopatias, caracterizada por agravamento do quadro clínico e mortalidade. Neste contexto, a terapêutica medicamentosa não consegue, em muitos casos, manter qualidade de vida adequada e sobrevida da pessoa, sendo o transplante cardíaco o recurso encontrado para melhorar a qualidade ou prolongar a vida ${ }^{(1,2)}$.

O transplante cardíaco é considerado o tratamento gold standard para pessoas selecionadas com doença cardíaca terminal, quando a terapia médica não for capaz de deter a progressão da patologia subjacente ${ }^{(3)}$.

Atualmente, a técnica mais usada nos centros de transplantação é a bicava (ortotópico), que consiste na desnervação do coração através da excisão completa da aurícula direita e do septo interauricular, mantendo a parede posterior da aurícula esquerda contendo as veias pulmonares. A desnervação cardíaca faz com que o controlo cardiorrespiratório (consumo máximo de $\mathrm{O}_{2}$ - $\left.\mathrm{VO}_{2 \mathrm{máx}}\right)$ e hemodinâmico (frequência cardíaca, débito cardíaco e pressão arterial) sejam inicialmente dependente do mecanismo de FrankStarling (lei da pré carga dependente do retorno venoso), das catecolaminas circulantes (influenciam o inotropismo) e da pós-carga (através das anteriores acarretam variações da fração de ejeção), pela ausência da estimulação simpática e parassimpática e pelo barorreflexo ${ }^{(4)}$. Assim, as pessoas transplantadas cardíacas apresentam menores valores de $\mathrm{VO}_{2 \text { máx }}$ (cerca de $70-80 \%$ do que o valor previsto para idade em relação às pessoas saudáveis), elevados valores de frequência cardíaca, pressão arterial e resistência vascular em repouso, sendo que em exercício verifica-se um aumento débil da frequência cardíaca e pressão arterial, associado a aumento da resistência vascular. Estas alterações, associadas aos efeitos da terapêutica imunossopressora, geram alterações cardiorrespiratórias e hemodinâmicas imediatas e a longo prazo, sendo que ao longo do tempo as pessoas transplantadas ao coração desenvolvem hipertensão arterial (95\%), dislipidémia (81\%), vasculopatia (50\%), insuficiência renal (33\%) e diabetes mellitus (32\%), sendo de suma importância a integração em programas de reabilitação nas suas diferentes fases ${ }^{(5)}$.

A reabilitação cardíaca é um processo de intervenção multifatorial, através do qual o indivíduo mantém ou recupera a sua condição física, psicológica, social e laboral de forma satisfatória, após um evento cardíaco ou no contexto de doença cardíaca crónica. Esta assenta na prática de exercício físico adaptado e na mudança de comportamentos, orientada para desencadear mudanças benéficas no estilo de vida, reduzir e controlar os fatores de risco, intervir em fatores psicológicos, com o objetivo de reverter ou atrasar a progressão da doença cardiovascular subjacente ${ }^{(6)}$.

O desafio para reabilitação cardíaca inicia-se no pré transplante sendo que, idealmente, todos os candidatos deveriam ser submetidos a reabilitação cardíaca adaptada ao seu status e causa de insuficiência cardíaca, facilitando à posteriori a sua recuperação pós transplante ${ }^{(7)}$. 
Apesar dos seus benefícios conhecidos menos de $20 \%$ destas pessoas participam em programas de reabilitação cardíaca no mundo ${ }^{(8)}$, não existindo referência em Portugal de nenhum programa estruturado de reabilitação cardíaca dirigido a pessoas transplantadas. Durante décadas, foram aplicadas restrições ao exercício às pessoas transplantadas ao coração, pois acreditava-se que o coração transplantado permanecia desnervado, com incompetência cronotrópica e, por isso, seria de risco a integração em programas de exercício. No entanto, existe agora ampla evidência de que o treino físico é bem tolerado e que este pode potenciar a reinervação e o controlo nervoso autónomo ${ }^{(2)}$, existindo um enfoque no específico no exercício físico.

Quadro 1 - Resumo da evidência científica da reabilitação cardíaca em transplantados cardíacos(9)

\begin{tabular}{|c|c|}
\hline Classe I & $\begin{array}{l}\text { Condições em que há evidências e/ou consenso de que dado } \\
\text { procedimento é eficaz ou útil }\end{array}$ \\
\hline \multicolumn{2}{|c|}{ 1. Reabilitação física precoce } \\
\hline \multicolumn{2}{|c|}{ 2. Atividade física aeróbia } \\
\hline \multicolumn{2}{|c|}{ 3. Atividade física com exercício resistido } \\
\hline \multicolumn{2}{|c|}{ 4. Programa de atividade supervisionado } \\
\hline \multicolumn{2}{|c|}{ 5. Teste de esforço, de preferência por teste cardiopulmonar } \\
\hline Classe II & $\begin{array}{l}\text { Condição em que há evidências divergentes de opinião a } \\
\text { respeito da utilidade e eficácia de tratamento e/ou } \\
\text { procedimento }\end{array}$ \\
\hline \multicolumn{2}{|c|}{ A - Evidência ou opinião que favorece a utilização do tratamento } \\
\hline \multicolumn{2}{|c|}{ 1. Programa de atividade física não supervisionado } \\
\hline \multicolumn{2}{|c|}{ 2. Atividade física em piscina aquecida } \\
\hline \multicolumn{2}{|c|}{ 3. Atividades recreativas } \\
\hline \multicolumn{2}{|c|}{ B - Evidência ou opinião menos estabelecida } \\
\hline \multicolumn{2}{|c|}{ 1. Participação em jogos competitivos sem supervisão } \\
\hline \multicolumn{2}{|c|}{ 2. Atividade física sem supervisão } \\
\hline Classe III & $\begin{array}{l}\text { Condição em que há evidência e/ou consenso de que o } \\
\text { procedimento/tratamento não é útil e em alguns casos pode } \\
\text { ser nocivo }\end{array}$ \\
\hline \multicolumn{2}{|c|}{ 1. Instabilidade hemodinâmica } \\
\hline \multicolumn{2}{|c|}{ 2. Episódio de rejeição leve a grave } \\
\hline \multicolumn{2}{|c|}{ 3. Processo de infeção } \\
\hline 4. Li & ortopédica e/ou neurológica que impossibilite \\
\hline
\end{tabular}

Os programas de reabilitação cardíaca em transplantação cardíaca devem incluir orientação nutricional, gestão do regime medicamentoso, intervenção psicológica na pessoa doente e plano de exercício/atividade física(10-13). Deve-se, no entanto, reforçar que o exercício físico regular tem tido um papel recente importante na terapêutica não medicamentosa das pessoas transplantadas, devendo ser iniciado o mais precocemente possível, na fase hospitalar, dando prosseguimento pós-alta, para que possam retornar a um estilo de vida semelhante ao que tinham antes da doença, permitindo convívio social satisfatório, com vida ativa e produtiva ${ }^{(8)}$. Em relação às intervenções definidas a serem executadas pela pessoa pós-transplante cardíaco, estas são diferentes conforme a fase e o local em que são efetuadas e devem ser adaptadas à situação específica de cada transplantado. Entende-se que os ganhos em saúde serão tanto maiores se o programa de RC for composto pelas 3 fases. De início no internamento (após a extubação) ${ }^{(12-15)}$, passando para a fase II pós alta, com duração de cerca de 3 meses (hospitalar, domiciliário ou comunitário), com supervisão periódica da pessoa doente pela equipa de reabilitação cardíaca ${ }^{(12,13)}$ e culminando na fase III, de manutenção prolongando-se pela vida toda da pessoa, na maioria das vezes recomendada em Home-based, pela incremento da adesão do doente ${ }^{(16)}$.

A pessoa transplantada ao coração vivencia um particular momento de transição sendo que, normalmente, a família será um suporte - físico, emocional, financeiro, entre outros - e numa fase inicial será o único apoio pela necessidade de isolamento pela situação clínica. 0 experienciar de uma situação limite associada ao risco de vida, é vivida de forma única, quer pela pessoa doente quer pela família. A pessoa doente só vivencia a gravidade da sua situação saúde/doença, muitas vezes num período de recuperação, enquanto a família experimenta intensamente todas as alterações e oscilações ao longo de todo o processo saúde/doença do doente ${ }^{(17)}$. Acresce ainda que, previamente, doentes e família experienciaram um período mais ou menos longo, de uma condição imprevisível e limitadora de vida, a insuficiência cardíaca. Esta condição clínica implica, na maioria das vezes, diferentes níveis de dependência, de défice na eficácia na autovigilância, na adesão ao regime terapêutico na sua globalidade e no exercício em especial, o que limitará na maioria das vezes por si só o pós-transplante. Desta forma, é importante o entendimento sobre o papel de cuidador que pode ser um fator de stress crónico e pode experimentar tristeza ${ }^{(18)}$.

Os cuidadores fornecem apoio às pessoas que vivem com condições de longo prazo existindo escassez de evidências das intervenções que apoiem os cuidadores no seu papel neste contexto específico. Não existindo evidência sobre a intervenção da RC no contexto da família da pessoa transplantada podemos de alguma forma ter como referência as evidências relacionadas com a família da pessoa com IC, status patológico prévio à transplantação cardíaca. Os cuidadores identificam 3 necessidades distintas: 
(1) apoiar a gestão da nova condição de saúde, que inclui lidar com a variabilidade dos sintomas/sinais, compreender e gerir medicamentos, fornecer apoio emocional, promover o exercício e a atividade física; (2) desenvolver o papel de cuidador, incluindo a comunicação com profissionais de saúde, gerir o seu próprio bem-estar e (3) desenvolver estratégias de apoio social, voluntário e serviços, reconhecendo que o futuro a longo prazo pode ser incerto ${ }^{(15)}$. Neste sentido, é necessário capacitar o prestador de cuidados e/ou família dotando-a de recursos, tornando-os mais aptos e resilientes na gestão da doença do seu familiar ${ }^{(19)}$. Para além da perspetiva do cuidador/família como parceiro de cuidados, não pode num contexto de doença cardiovascular ser esquecida a saúde do próprio cuidador, isto porque muitas vezes a patologia cardíaca conducente à necessidade de transplante da pessoa tem na sua génese diversos fatores de risco cardiovascular que são partilhados com os seus conviventes, colocando-os também em risco de desenvolver patologia.

A Enfermagem de Reabilitação (ER) é considerada uma área de intervenção especializada que engloba um corpo de conhecimento e procedimentos específicos ${ }^{(20)}$. 0 enfermeiro especialista em enfermagem de reabilitação (EER) será fundamental neste fenómeno de transição, uma vez que apresenta um conhecimento aprofundado no diagnóstico e na intervencão precoce, na promocão da qualidade de vida, na maximização da funcionalidade, no autocuidado e na prevenção de complicaçõ̂es, evitando as incapacidades ou minimizando as mesmas Regulamento dos padrões de qualidade dos cuidados ${ }^{(20,21)}$. Neste contexto será necessária mestria entre a transição de diferentes naturezas, com diferentes agentes facilitadores/dificultadores e com padrões de resposta por vezes imprevisíveis. No seu perfil de competências o EER promove intervenções preventivas, quer para assegurar as capacidades funcionais das pessoas doentes, quer para evitar mais incapacidades, previne complicações e defende e promove o direito à qualidade de vida, à socialização e à dignidade, assumindo, deste modo, o papel de educador, gestor de casos, conselheiro, capacitador, facilitador e membro da equipa ${ }^{(20-22)}$. Estes têm o dever e competência de ensinar, instruir, treinar, orientar e motivar quer as pessoas doentes nas atividades de vida diária, para níveis máximos de interdependência, quer os respetivos familiares nos processos de reabilitação, promovendo o autocuidado ${ }^{(21,22)}$. 0 saber e as competências do EER possibilitam, junto da pessoa com incapacidade ou deficiência, a gestão de situações complexas de saúde, a intervenção ao longo do ciclo de vida, a procura contínua dos melhores resultados para a pessoa doenté/família, a implementação de parcerias com o pessoa, a família ou a comunidade durante o processo de reabilitação e a coordenação de planos de cuidados interdisciplinares ${ }^{(18)}$.

Os programas de reabilitação cardíaca $(\mathrm{RC})$, nas suas diferentes fases, permitem ao enfermeiro especialista em enfermagem de reabilitação (EEER) ter uma intervenção privilegiada no acompanhamento e cuidado à pessoa transplantada ao coração, desde a fase pré-operatória, no período de recuperação pós-operatório imediato e tardio. Contribui para a promoção da autonomia e autocuidado da pessoa e sua família, na adaptação à nova condição de saúde e no aumento da sua qualidade de vida, intervindo nas diversas alterações psicológicas e fisiológicas decorrentes do transplante. Os EER têm formação para planificacão de exercício físico, para que as pessoas sejam capacitadas a manterem-se ativas após o internamento, que corresponde à fase Î da reabilitação cardíaca e após um período de 8 semanas de reabilitação em ambulatório - fase II, assim como na fase III, quer com programas Home-based ou Center based, devendo estar presente nas 3 fases da RC com a pessoa, independentemente da idade e sua família ${ }^{(12,13,23)}$.

A excelência da enfermagem de reabilitação, com intervenção baseada na evidência, origina ganhos em saúde(20), ganhos que devem expressar a melhoria dos resultados e traduzirem-se por ganhos em anos de vida, pela redução de episódios de doença ou encurtamento da sua duração, pela diminuição das situações de incapacidade temporária ou permanente, pelo aumento da funcionalidade física e psicossocial e, ainda, pela redução do sofrimento evitável e melhoria da qualidade de vida relacionada ou condicionada pela saúde ${ }^{(24)}$. Os ganhos em saúde sensíveis aos cuidados de enfermagem demonstram o estado, comportamento ou perceção variável e mensurável de um utente ou seu convivente significativo, que è amplamente influenciado pelas intervenções de enfermagem que dependem da conceção do enfermeiro, de acordo com os mandatos sociais com que a enfermagem está investida $^{(25)}$.

\section{METODOLOGIA}

A procura do conhecimento enquanto suporte para uma prática de cuidados de enfermagem de reabilitação baseada na evidência é fundamental ${ }^{(22)}$, sendo o objetivo major, neste contexto, identificar em que domínios é que os enfermeiros especialistas de reabilitação (EER) poderão intervir de forma a desenvolver ganhos em saúde de pessoas transplantadas cardíacas e seu cuidador/familia. Torna-se fundamental evidenciar esses ganhos em saúde, sendo que será usado a The Quality Health Outcomes Model de Doran ${ }^{(26)}$, que apesar de abordar estrutura, processo e resultados, será nesta última componente, que será analisado neste trabalho. Como forma de estruturar a mensuração dos ganhos em saúde através dos resultados, serão definidas 6 categorias, adaptadas de Irvine, Sidani, \& Hall (1998): (i) prevenção de eventos adversos; (ii) resultados clínicos, incluindo controlo de sintomas; (iii) conhecimento da pessoa sobre a doença, seus tratamentos e gestão associada; (iv) resultados funcionais de saúde, incluindo capacidade física, social, cognitiva e mental e habilidades de autocuidado; (v) satisfação da pessoa e cuidador com o cuidado; e (vi) resultados económicos e ainda que não incorporados no modelo original também a necessidade de cuidados de saúde e mortalidade ${ }^{(27)}$.

O projeto Enfermagem de Reabilitação em Transplante Cardíaco (ERtxC) apresenta como pergunta de investigação "Quais os ganhos em saúde de um programa de Reabilitação cardíaca para a pessoa transplantada e sua família?".

Para responder à pergunta de investigação foram definidos os seguintes objetivos:

Desenvolver um programa de reabilitação cardíaca nas diferentes fases para a pessoa transplantada ao coração;

Desenvolver programa de reabilitação cardíaca para o cuidador da pessoa transplantada ao coração;

Mensurar os ganhos em saúde inerentes ao programa de reabilitação cardíaca na pessoa transplantada ao coração;

Mensurar os ganhos em saúde inerentes ao programa de reabilitação cardíaca ao cuidador da pessoa transplantada ao coração;

Desenvolver indicadores de saúde resultado do programa de reabilitação cardíaca para pessoas e família. 
Perante os focos desta investigação considera-se que terão de ser efetuados de forma concorrente 2 estudos, um direcionado à reabilitação cardíaca da pessoa transplantada e outro com enfoque na reabilitação cardíaca do cuidador/família, sendo eles próprios divididos em metodologias diferentes, dependendo da fase em que se encontram.

Pretende-se a implementação e avaliação dos resultados do programa ERtxC que se pressupõe dividido em 4 fases distintas (Figura 1).

\begin{tabular}{|c|c|}
\hline \multicolumn{2}{|c|}{ Nursing Rehabilitation for Heart Transplant (NR4HTx) } \\
\hline $\begin{array}{l}\text { Hospital Rehabilitation Nursing for Heart Transplant } \\
\qquad(H R \tilde{N} 4 H T x)\end{array}$ & $\begin{array}{c}\text { Programa de reabilitação cardiaca fase } 1 \text { para pessoas } \\
\text { transplantadas ao coração (internamento) }\end{array}$ \\
\hline $\begin{array}{l}\text { Ambulatory Rehabilitation Nursingfor Heart } \\
\text { Transplant (ARN4HTx) }\end{array}$ & $\begin{array}{c}\text { Programa de reabilitação cardiaca fase } 2 \text { para pessoas } \\
\text { transplantadas ao coração (ambulatório-até } 2 \text { meses } \\
\text { pós transplante) }\end{array}$ \\
\hline $\begin{array}{l}\text { Physical Activity for Heart Transplant at Home } \\
\qquad(P A 4 T \times H)\end{array}$ & $\begin{array}{c}\text { Programa de reabilitação cardiaca fase } 3 \text { para pessoas } \\
\text { transplantadas ao coração (home based) }\end{array}$ \\
\hline $\begin{array}{l}\text { Cardiac Rehabilitation for heart transplant caregivers } \\
\qquad(C R H T x C)\end{array}$ & $\begin{array}{c}\text { Programa de reabilitação cardiaca para os } \\
\text { cuidadores/familia de pessoas transplantadas ao } \\
\text { coração }\end{array}$ \\
\hline
\end{tabular}

Figura 1 - Fases do programa ERtxC

Atendendo que a população deste estudo são as pessoas transplantadas ao coração em Portugal, a amostra será obtida no Centro de referência de transplantação cardíaca com maior atividade nacional, sendo incluídas todas as pessoas transplantadas ao coração e cuidadores/família com mais de 18 anos de idade, que reúnam condições clínicas (físicas e psíquicas) e que facultem o seu consentimento.

Para mensurar os ganhos em saúde decorrentes da implementação pretende-se usar como metodologia inicialmente o relato de caso, de forma a verificar a sua aplicabilidade e limitações e, posteriormente, o estudo clínico randomizado(28-30). Este tipo de estudo permite a comparação entre grupos sendo que serão definidos: Grupo de Intervenção (GT - Programa de reabilitação cardíaca estruturado) e Grupo de controlo (GC) - receberá intervenção de reabilitação até agora definida (Figura 2).

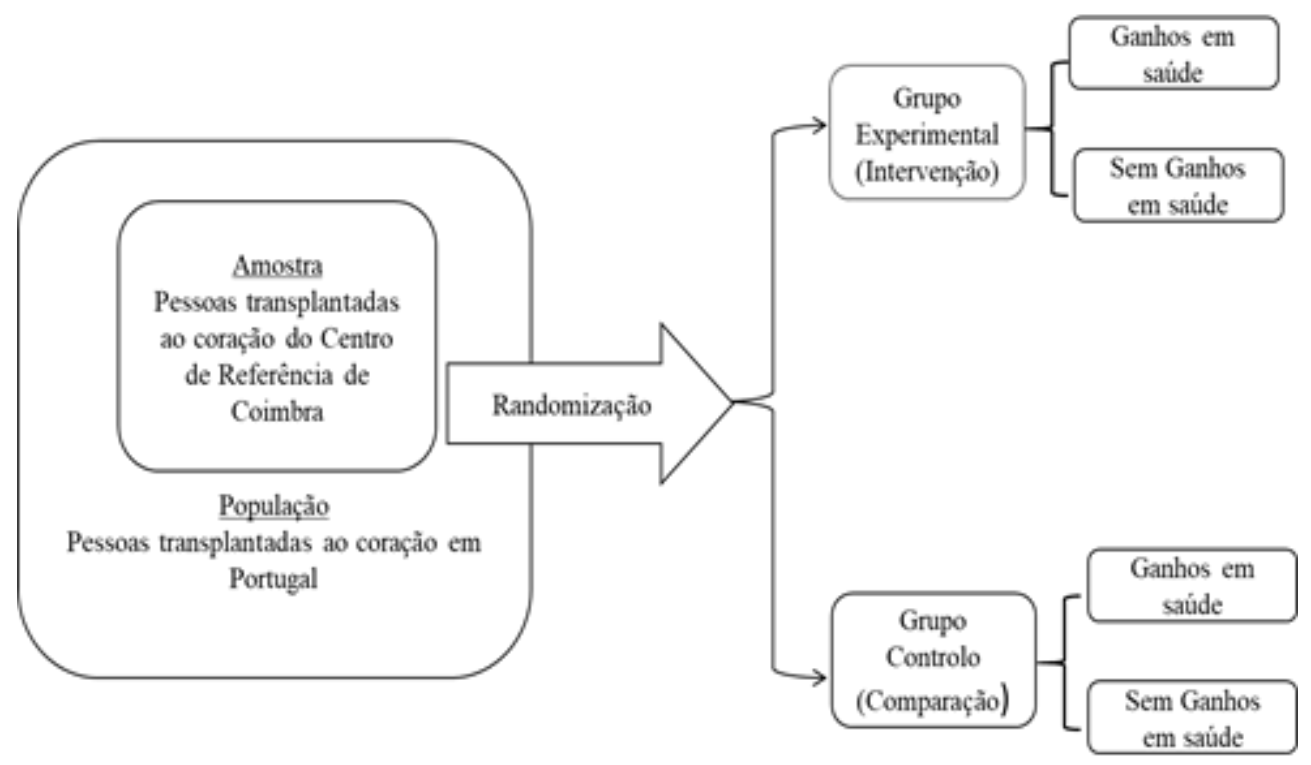

Figura 2-Representação do desenho de estudo

Todas pessoas que reúnam os critérios de inclusão serão randomizados (1:1), recorrendo ao site www.randomizer.org. Se o resultado da randomização for 1 , significa que a pessoa doente será incluído no grupo teste (GT), se o resultado for 2 , a pessoa doente será incluído no grupo controlo (GC).

Antes do início proceder-se-á ao registo no Clinical trials, de forma a garantir que pessoas e profissionais têm acesso à informação do estudo, impulsionando o conhecimento da ciência e a cooperação entre grupos de pesquisa. 
Em relação ao estudo do cuidador/família optar-se-á por efetuar uma scoping review de forma a mapear a mais adequada evidência científica para, em seguida, estruturar um programa de intervenção dirigido ao cuidador/família. Esta será efetuada utilizando a metodologia definida pelo Joanna Briggs Institute ${ }^{(31,32)}$. Será desenvolvido um programa de reabilitação nas diferentes fases para cuidador/família, recorrendo-se a um estudo exploratório ${ }^{(28,29)}$.

A junção dos 2 estudos pode ser representada no seguinte fluxograma representado pela Figura 3.

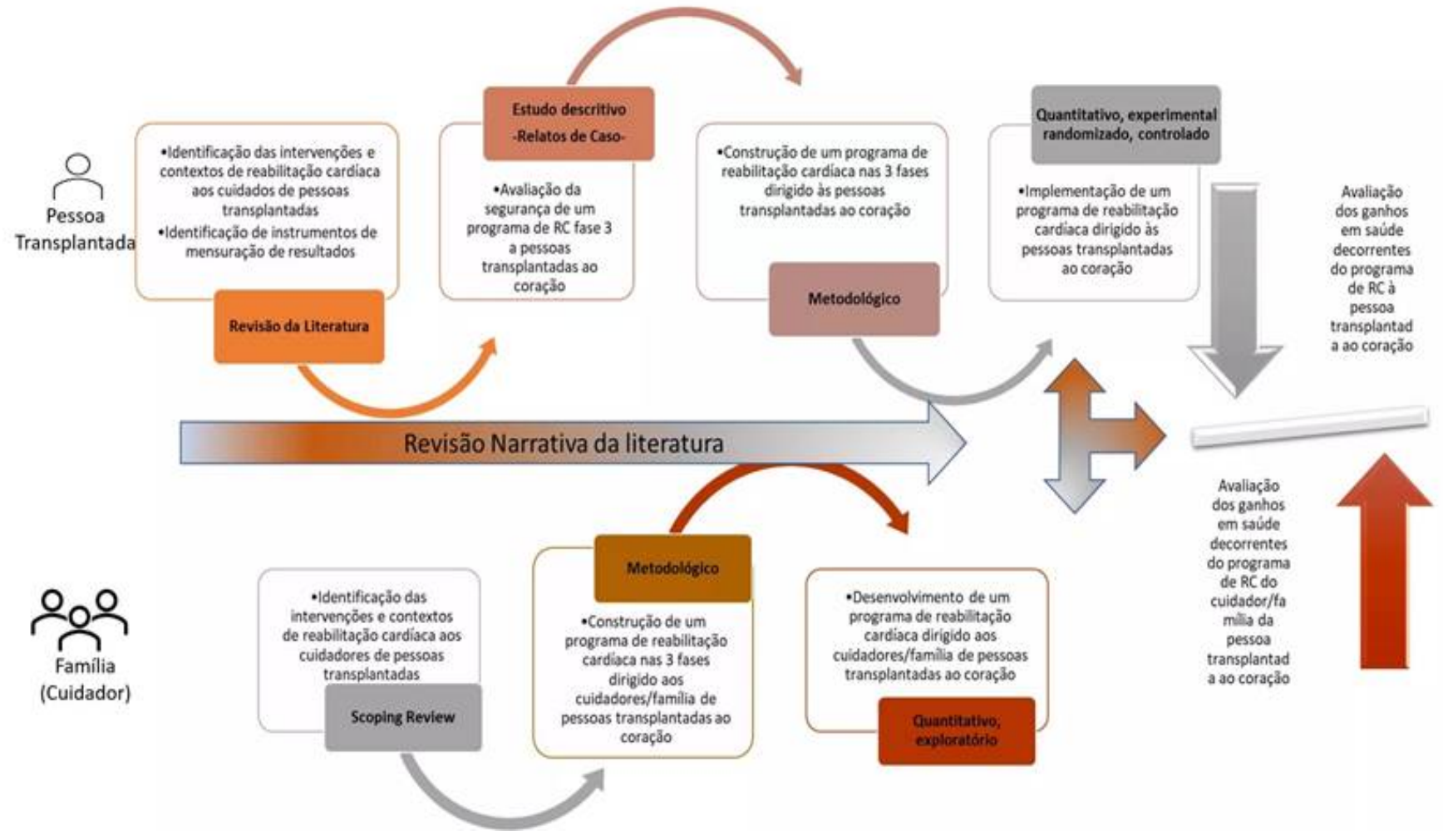

Figura 3 - Fluxograma dos dois estudos

Em relação à estrutura de implementação após a primeira revisão da literatura e opções metodológicas prevê-se o seguinte percurso:

\section{Intervenção:}

1. Avaliação Inicial - Consulta Pré-transplante - Pessoa referenciada para transplante cardíaco e Família (consentimento para integração no estudo)

2. Plano de Intervenção

\begin{tabular}{|l|l|}
\hline População alvo & $\begin{array}{l}\text { Pessoas Transplantadas cardíacos e } \\
\text { Cuidador/Família }\end{array}$ \\
\hline & $\begin{array}{l}\text { Escala de Qualidade de Vida (EuroQol) } \\
\text { Teste de marcha de 6 minutos } \\
\text { Escala de Borg modificada } \\
\text { Instrumentos de avaliação da } \\
\text { pessoa transplantada }\end{array}$ \\
& $\begin{array}{l}\text { Estratificação do risco } \\
\text { Avaliação da força (dinamómetro) } \\
\text { Escala de London Chest Activities of Daily } \\
\text { Living (LCADL) } \\
\text { Self-care adherence (I. O. Kim, 2006) }\end{array}$ \\
\hline
\end{tabular}




\begin{tabular}{|c|c|}
\hline $\begin{array}{l}\text { Instrumentos de avaliação do } \\
\text { Cuidador/ família }\end{array}$ & $\begin{array}{l}\text { Family Caregiver Quality of Life Scale } \\
\text { questionnaire (FamQol) } \\
\text { Caregiver Contribution to Self-Care of } \\
\text { Failure Index (CC-SCHFI) }\end{array}$ \\
\hline $\begin{array}{l}\text { Tipologia de exercícios (programa } \\
\text { divide-se em diferentes estadios } \\
\text { sendo prescrito de forma individual } \\
\text { com base no FITT-VP Frequência, } \\
\text { Intensidade, Tempo, Tipo-Volume, } \\
\text { Progressão) }\end{array}$ & $\begin{array}{l}\text { Exercícios Respiratórios } \\
\text { Aeróbio: } \\
\text { Pedaleira } \\
\text { Marcha } \\
\text { Subir e descer escadas } \\
\text { Bicicleta estática } \\
\text { Passadeira } \\
\text { Força: } \\
\text { Membros superiores e inferiores } \\
\text { Aquecimento/Relaxamento } \\
\text { Exercícios de Alongamento antes e após o } \\
\text { treino }\end{array}$ \\
\hline Critérios de segurança utilizados & $\begin{array}{l}\text { Variação da frequência cardíaca em exercício } \\
\text { Perceção subjetiva de esforço } \\
\text { Tensão arterial e Saturação de } 02 \\
\text { Vigilância de Ritmo por monitor (Telemetria) } \\
\text { Sudorese - presença } \\
\text { Tonturas }\end{array}$ \\
\hline $\begin{array}{l}\text { Parâmetros de execução de } \\
\text { exercício físico analisados }\end{array}$ & $\begin{array}{l}\mathrm{N}^{\circ} \text { de voltas na pedaleira } \\
\mathrm{N}^{\circ} \text { de metros } \\
\mathrm{N}^{\circ} \text { de degraus } \\
\mathrm{N} .^{\circ} \text { de minutos } \\
\text { Valor de resistência da bicicleta } \\
\text { Incremento de resistência }\end{array}$ \\
\hline Duração de cada sessão & $\begin{array}{l}\text { Fase I -Entre } 5 \text { a } 30 \text { minutos (em função do } \\
\text { estadio) } \\
\text { Fase II - 20-40 minutos } \\
\text { Fase III- 30-45 minutos }\end{array}$ \\
\hline $\begin{array}{l}\text { Frequência de aplicação do } \\
\text { programa }\end{array}$ & $\begin{array}{l}\text { Fase I - Idealmente bidiário } \\
\text { Fase II - Diário } \\
\text { Fase III - } 3 \text { a } 4 \text { vezes por semana }\end{array}$ \\
\hline
\end{tabular}




\begin{tabular}{|l|l|}
\hline Intervenção Educacional & Fatores de risco cardiovascular \\
& Gestão do regime terapêutico \\
\hline Intervenção Familiar & $\begin{array}{l}\text { Construção de Jogo de tabuleiro dirigido ao } \\
\text { cuidador }\end{array}$ \\
& Ensino sobre Suporte básico de vida \\
Controlo de fatores de risco \\
\hline
\end{tabular}

\section{Avaliação dos Resultados}

Utilizar-se-ão os mesmos instrumentos no final de cada etapa do programa de RC, no caso da pessoa transplantada ao coração e no final de cada fase, no caso do cuidador, será construída uma grelha de resultados sensíveis aos cuidados de enfermagem como definido por Doran.

Este projeto de intervenção cumprirá com todos os pressupostos éticos, foi submetido a avaliação por parte de uma Comissão de Ética e aguarda o seu parecer para ter início.

0 registo em bruto dos dados será feito em folha do programa Microsoft Excel criada especificamente para este efeito. A análise dos dados resultantes desta intervenção será feita através de estatística descritiva e inferencial, com os testes adequados, através do programa IBM ${ }^{\circledR}$ SPSS $^{\circledR} 23$, de forma a poderem ser comparados os grupos mas também os diferentes momentos de avaliação.

\section{DISCUSSÃO/RESULTADOS ESPERADOS}

Ao longo dos anos de 2021 e 2022 prevê-se a construção e implementação do programa Enfermagem de Reabilitação em Transplante Cardíaco (ERtxC), nas suas diferentes fases. Estima-se que o programa possa ser acedido por 150 pessoas transplantadas cardíacas e respetivas famílias/cuidador.

\section{CONCLUSÃO}

No final do ano de 2022 estima-se ter chegado ao final deste programa Enfermagem de Reabilitação em Transplante Cardíaco (ERtxC), avaliando-se os ganhos em saúde decorrentes do programa de RC à pessoa transplantada ao coração e respetivas famílias/cuidador. Após o ano de 2022 e após validação deste programa, tentar-se-á criar em definitivo esta oferta de forma sistemática para todas pessoas transplantadas ao coração, e respetivas famílias, nas diferentes unidades hospitalares do País que fazem o seguimento destas pessoas.

\section{DIVULGAÇÕES ÉTICAS}

Contribuição do(s) autor(es):

Conceptualização: ML, ES, AN

Metodologia: ML, GC, AN

Validação: MM, EL, MC, AN

Análise formal: MM, MC, GC

Preparação do rascunho original: ML, MC, ES

Redação e edição: $M L$, AN

Revisão: ML, MM

Todos os autores leram e concordaram com a versão publicada do manuscrito.

\section{Financiamento:}

Este trabalho não recebeu nenhuma contribuição financeira ou bolsa. 
A aguardar parecer da Comissão de Ética.

Declaração de consentimento informado:

Não se aplica.

Agradecimentos:

Os autores agradecem a todos quantos contribuem para o desenvolvimento deste trabalho.

Conflitos de interesse:

Os autores não declaram nenhum conflito de interesses.

Proveniência e revisão por pares:

Não comissionado; revisto externamente por pares.

\section{Referências}

1. Lund LH, Khush KK, Cherikh WS, Goldfarb S, Kucheryavaya AY, Levvey BJ, et al. The Registry of the International Society for Heart and Lung Transplantation: Thirty-fourth Adult Heart Transplantation Report-2017; Focus Theme: Allograft ischemic time. J Hear Lung Transplant. 2017 Oct;36(10):1037-46. Available from: https://doi.org/10.1016/j.healun.2017.07.019

2. Nygaard S, Christensen AH, Rolid K, Nytrøen K, Gullestad L, Fiane A, et al. Autonomic cardiovascular control changes in recent heart transplant recipients lead to physiological limitations in response to orthostatic challenge and isometric exercise. Eur $\mathrm{J}$ Appl Physiol. 2019 Aug 12; Available from: https://doi.org/10.1007/s00421-019-04207-5

3. Anderson L, Nguyen TT, Dall CH, Burgess L, Bridges C, Taylor RS. Exercise-based cardiac rehabilitation in heart transplant recipients. Cochrane Database Syst Rev. 2017 Apr 4; Available from: https://doi.org/10.1002/14651858.CD012264.pub2

4. Loureiro M de F de S. Reabilitação e transplante cardíaco: revisão sistemática da literatura [Internet]. Instituto Politécnico de Bragança; 2015.

5. Perrier-Melo RJ, Figueira FAM dos S, Guimarães GV, Costa M da C. High-Intensity Interval Training in Heart Transplant Recipients: A Systematic Review with Meta-Analysis. Arq Bras Cardiol. 2018;110(2):188-94. Available from: https://doi.org/10.5935/abc.20180017

6. Lancellotti P, Ancion A, Piérard L. [Cardiac rehabilitation, state of the art 2017]. Rev Med Liege. 2017 Nov;72(11):481-7.

7. ACSM. ACSM's guideline for exercise testing and prescription. Tenth Edit. American College of Sports Medicine; 2018.501 p.

8. Bachmann JM, Shah AS, Duncan MS, Greevy RA, Graves AJ, Ni S, et al. Cardiac rehabilitation and readmissions after heart transplantation. J Hear Lung Transplant. 2018 Apr;37(4):467-76. Available from: https://doi.org/10.1016/j.healun.2017.05.017

9. Fogaça D, Goés GG de, Fuhro MI, Mariante P, Frasson R, Monteiro MB, et al. O papel da reabilitação física após o transplante cardíaco: uma revisão da literatura. EFDeportes. 2012;(171).

10. Uithoven K, Smith J, Medina-Inojosa J, Squires R, Van Iterson E, Olson T. Clinical and Rehabilitative Predictors of Peak Oxygen Uptake Following Cardiac Transplantation. J Clin Med. 2019 Jan 19;8(1):119. Available from: https://doi.org/10.3390/jcm8010119

11. Chen AC, Rosenthal DN, Couch SC, Berry S, Stauffer KJ, Brabender J, et al. Healthy hearts in pediatric heart transplant patients with an exercise and diet intervention via live video conferencing-Design and rationale. Pediatr Transplant. 2019;23(1):e13316. Available from: https://doi.org/10.1111/petr.13316

12. Ordem dos Enfermeiros. Guia Orientador de Boa Prática em Enfermagem de Reabilitação: Reabilitação Cardíaca [Internet]. 2020.

13. Novo A, Delgado B, Mendes E, Lopes I, Preto L, Loureiro M. Reabilitação cardíaca - evidência e fundamentos para a prática. Loures: Lusodidacta; 2020.

14. Kawauchi TS, Almeida PO de, Lucy KR, Bocchi EA, Feltrim MIZ, Nozawa E. Randomized and comparative study between two intra-hospital exercise programs for heart transplant patients. Rev Bras Cir Cardiovasc. 2013;28(3):338-46. Available from: https://doi.org/10.5935/1678-9741.20130053

15. Polastri M, Savini C, Grigioni F. Calf cramps in a heart transplant patient during the postoperative course: a case report. Int J Ther Rehabil. 2013 Jan 2;20(1):55-7. Available from: https://doi.org/10.12968/ijtr.2013.20.1.55

16. Thomas RJ, Beatty AL, Beckie TM, Brewer LC, Brown TM, Forman DE, et al. Home-Based Cardiac Rehabilitation: A Scientific Statement From the American Association of Cardiovascular and Pulmonary Rehabilitation, the American Heart Association, and the American College of Cardiology. Circulation. 2019 Jul 2;140(1). Available from: https://doi.org/10.1161/CIR.0000000000000663

17. Sá FLFRG de, Botelho MAR, Henriques MA. Cuidar da família da pessoa em situação crítica. Pensar Enferm. 2015;19(1):31-46.

18. Hoeman S. Enfermagem de reabilitação: Prevenção, intervenção e resultados esperados. Loures: Lusociência. $2011 ; 556$.

19. Wingham J, Frost J, Britten N, Greaves C, Abraham C, Warren FC, et al. Caregiver outcomes of the REACH-HF multicentre randomized controlled trial of home-based rehabilitation for heart failure with reduced ejection fraction. Eur $\mathrm{J}$ Cardiovasc Nurs. 2019 Oct 22;18(7):611-20. Available from: https://doi.org/10.1177/1474515119850011 
20. Mesa do Colégio de Especialidade de Enfermagem de Reabilitação da Ordem dos Enfermeiros. Regulamento dos Padrões de Qualidade dos Cuidados Especializados em Enfermagem em Enfermagem de Reabilitação - Regulamento n.o 350/2015 Regulamento - Diário da República, 2.a série - N.o 119 - 22 de junho de 2015. 2015.

21. Mesa do Colégio de Especialidade de Enfermagem de Reabilitação da Ordem dos Enfermeiros. Regulamento das Competências Específicas do Enfermeiro Especialista em Enfermagem de Reabilitação - Regulamento n.o 125/2011 - Diário da República 2.a série - N.o 35 - 18 de Fevereiro de 2011 [Internet]. 2011.

22. Ordem dos Enfermeiros. Regulamento das Competências Comuns do Enfermeiro Especialista [Internet]. 2011.

23. Loureiro M, Duarte J, Sola E, Martins MM, Novo A. Programa de reabilitação cardíaca home-based da pessoa transplantada ao coração: relato de caso. Rev Port Enferm Reabil. 2020 Oct 27;3(S1):42-9. Available from: https://doi.org/10.33194/rper.2020.v3.s1.5.5771

24. Direção Geral da Saúdeisb. Plano Nacional de Saúde - Revisão e extensão a 2020 [Internet]. 2015.

25. Ordem dos Enfermeiros. Core de indicadores por categoria de enunciados descritivos dos Padrões de Qualidade dos Cuidados de Enfermagem de Reabilitação (PQCER) [Internet]. 2015.

26. Amaral A. A efectividade dos cuidados de enfermagem: modelos de análise. Rev Investig em Enferm. 2010;21:96-105.

27. Lukewich JA, Tranmer JE, Kirkland MC, Walsh AJ. Exploring the utility of the Nursing Role Effectiveness Model in evaluating nursing contributions in primary health care: A scoping review. Nurs Open. 2019 Jul 16;6(3):685-97. Available from: https://doi.org/10.1002/nop2.281

28. Souza RF. O que é um estudo clínico randomizado? Med (Ribeirão Preto). 2009;42(1):3-8.

29. Ribeiro GM. Novo manual de investigação. Lisboa: Contraponto. 2018;

30. Vilelas J. Investigação: O processo de Construção do Conhecimento. Lisboa: Edições Sílabo. Teses, Diss e provas académicas. 2009;

31. Peters M, Godfrey C, Mclnerney P, Soares C, Khalil H, Parker D. Methodology for JBI scoping reviews. The Joanna Briggs Institute reviewers' manual. Adelaide (Australia): The Joanna Briggs Institute; 2015. p. 1-24.

32. Peters MDJ, Godfrey CM, Khalil H, Mclnerney P, Parker D, Soares CB. Guidance for conducting systematic scoping reviews. Int J Evid Based Healthc. 2015 Sep;13(3):141-6. Available from: https: //doi.org/10.1097/XEB.0000000000000050

Este trabalho encontra-se publicado com a Licença Internacional Creative Commons Atribuição-NãoComercial-SemDerivações 4.0.

Direitos de Autor (c) 2021 Revista Portuguesa de Enfermagem de Reabilitação 